\title{
New approach to the adiabaticity concepts in the collective nuclear motion: Impact for the collective-inertia tensor and comparisons with experiment
}

\author{
D. Rouvel ${ }^{1,2, *}$ and J. Dudek ${ }^{2,3, \dagger}$ \\ ${ }^{1}$ Lycée Kléber, 25 place de Bordeaux, F-67000 Strasbourg, France \\ ${ }^{2}$ IPHC/DRS and Université de Strasbourg, 23 rue du Loess, B.P. 28, F-67037 Strasbourg Cedex 2, France \\ ${ }^{3}$ Institute of Physics, Marie Curie-Skłodowska University, PL-20031 Lublin, Poland
}

(Received 5 March 2018; published 19 April 2019)

\begin{abstract}
We propose a new derivation of the cranking-model expression for the nuclear collective inertia tensor, introducing explicitly the slow- and fast-motion timescales together with a parameter controlling the interplay between the two modes. The new cranking formula is free from the mass tensor divergencies originating from the nucleon-level crossings in the denominators of the first-order perturbation theory expressions and allows the exploration of the unrestricted deformation space without spurious mass tensor contributions caused by those divergencies. We apply the new formalism without extra parameter adjustments to the collective vibrations in ${ }^{208} \mathrm{~Pb}$.
\end{abstract}

DOI: 10.1103/PhysRevC.99.041303

As it is well known, the strong interactions acting among the nucleons are of a very short range so that nucleons in a nucleus can be seen as "touching each other." This allows introducing the notion of a nuclear surface whose shape can be identified with the nuclear shape. The equation of this surface can be described using a set of parameters, say $q \equiv\left\{q^{n} ; n=\right.$ $1,2, \ldots, d\}$, sometimes referred to as collective variables. They are particularly well adapted to studying various forms of the surface-vibrational (thus collective) motion.

In this Rapid Communication we present a new approach to calculating the nuclear collective inertia tensor in the $d$ dimensional curvilinear space of collective variables $q$ within the adiabatic cranking method. We introduce explicitly two timescales and derive a new factor-called temporizer, see Ref. [1] - in the modified expression for the inertia tensor. Its presence takes away the problem of singularities caused by the single-particle level crossings in the perturbation-theory mass tensor, a strongly undesired byproduct of the first-order perturbation theory. At the same time we approach the solution of the long-standing issue of the missing factor of the order of two, see Ref. [2], in the cranking expression for the inertia tensor.

The importance of including both the diagonal and nondiagonal components of the inertia tensor arises naturally in the description of the large-amplitude collective motion and in particular fission, see recent Ref. [3]. The notion of an

\footnotetext{
*david.rouvel@iphc.cnrs.fr

$\dagger$ jerzy.dudek@iphc.cnrs.fr
}

Published by the American Physical Society under the terms of the Creative Commons Attribution 4.0 International license. Further distribution of this work must maintain attribution to the author(s) and the published article's title, journal citation, and DOI. inertia tensor is a standard element in the microscopic nuclear structure approaches such as generator coordinate (GCM) or adiabatic time-dependent Hartree-Fock-Bogoliubov (ATDHFB), Refs. [4-6] and references therein. The starting point of the present formulation of the microscopic theory of the inertia tensor goes back to Ref. [7], see also Refs. [8-12], whereas discussion and extensive list of references related to the microscopic aspects of collective model can be found in, e.g., Refs. [13,14].

Let $\hat{x}_{k} \equiv\left\{\hat{\boldsymbol{r}}_{k}, \hat{\boldsymbol{p}}_{k}, \hat{\boldsymbol{s}}_{k}, \hat{\boldsymbol{t}}_{k}\right\}$ denote the ensemble of operators of position, linear momentum, spin, and isospin of a nucleon $k$, and let $\hat{x}=\left\{\hat{x}_{1}, \ldots, \hat{x}_{A}\right\}$ denote their full set. With the nuclear many-body Hamiltonian, $\hat{H}_{\mathrm{mb}}(\hat{X}, t)$, the corresponding Schrödinger equation can be formally written as

$$
\hat{H}_{\mathrm{mb}}(\hat{x}, t) \Psi_{\mathrm{mb}}(x, t)=i \hbar \frac{\partial \Psi_{\mathrm{mb}}}{\partial t}(x, t),
$$

where $\Psi_{\mathrm{mb}}(x, t)$ is the time-dependent multinucleon wave function.

Following Bohr and Mottelson let us introduce the concept of two energy scales: (i) the collective vibration-type energies, which are of the order of an $\mathrm{MeV}$, and (ii) the individualnucleonic energies, which can reach a few dozen MeV. Assuming the orders of magnitude for the typical collective vibration energy range as $0.5 \leqslant E_{\text {col }} \leqslant 2 \mathrm{MeV}$ and similarly for the individual-nucleonic motion in the vicinity of the typical Fermi energy as $30 \leqslant E_{\text {ind }} \leqslant 50 \mathrm{MeV}$, from the Planck relation, we obtain the two respective timescales: the one for the slow collective nuclear motion

$$
2 \times 10^{-21} \leqslant \tau_{\text {col }} \leqslant 8 \times 10^{-21} \mathrm{~s}
$$

and the other one for the quick individual nucleonic motion

$$
8.3 \times 10^{-23} \leqslant \tau_{\text {ind }} \leqslant 14 \times 10^{-23} \mathrm{~s} .
$$


The slow motion of the nuclear surface modifies the fast motion of each nucleon in a characteristic manner: its slowness allows nucleons to adapt their motion to the instantaneous mean field, the latter controlled by the ensemble of collective variables $q$ varying in time an order of magnitude slower.

Following this concept, the explicit dependence of the Hamiltonian on time can be replaced by an implicit dependence via collective variables, $q=q(t)$. Under these circumstances the adiabatic theorem of Refs. $[15,16]$ becomes applicable. It reads: "If the Hamiltonian of a system is dependent on time, the Schrödinger equation of motion (1) has in general no stationary solution. But in the limit when the change of the Hamiltonian is made infinitely slow, the system, when started from a stationary state of the Hamiltonian at $t_{0}$, passes through the corresponding stationary states for all $t$." Accordingly one may adapt the notation for the Hamiltonian $\hat{H}_{\mathrm{mb}}(\hat{x}, t) \rightarrow$ $\hat{H}_{\mathrm{mb}}^{\mathrm{ad}}(\hat{X} ; q)$ with $q=q(t)$ and the Schrödinger equation in (1) takes the form

$$
\hat{H}_{\mathrm{mb}}^{\mathrm{ad}}(\hat{x} ; q) \Psi_{\mathrm{mb}}^{\mathrm{ad}}(x, q, t)=i \hbar \frac{\partial \Psi_{\mathrm{mb}}^{\mathrm{ad}}}{\partial t}(x, q, t) .
$$

Since according to this formulation the time dependence of the Hamiltonian appears only through the slowly varying collective variables, it will be instructive to consider the time evolution of the Hamiltonian as a series of time-independent Hamiltonians at the progressing instants $t \rightarrow t_{1}, t_{2}, \ldots, t_{\ell}, \ldots$ with the corresponding collective coordinates $q_{1}, q_{2}, \ldots, q_{\ell}, \ldots$ and the static Hamiltonians, $\hat{H}_{\mathrm{mb}}^{\mathrm{ad}}\left(\hat{X} ; q_{1}\right), \hat{H}_{\mathrm{mb}}^{\mathrm{ad}}\left(\hat{X} ; q_{2}\right), \ldots, \hat{H}_{\mathrm{mb}}^{\mathrm{ad}}\left(\hat{X} ; q_{\ell}\right), \ldots .$. For each of those Hamiltonians we can write the induced stationary forms of the Schrödinger equation:

$$
\hat{H}_{\mathrm{mb}}^{\mathrm{ad}}\left(\hat{x} ; q_{\ell}\right) \Phi_{\mathrm{mb} ; k}\left(x ; q_{\ell}\right)=E_{\mathrm{mb} ; k}\left(q_{\ell}\right) \Phi_{\mathrm{mb} ; k}\left(x ; q_{\ell}\right) .
$$

In what follows we may simplify the notation by ignoring the evident index $\ell$ which becomes superfluous.

At this point we follow the standard approach of constructing $\Psi_{\mathrm{mb}}^{\mathrm{ad}}(x, q, t)$ as the linear combination of the above stationary solutions

$$
\Psi_{\mathrm{mb}}^{\mathrm{ad}}(x, q, t)=\sum_{k=0}^{N} C_{k}(t) \Phi_{\mathrm{mb} ; k}(x ; q) e^{i \varphi_{k}(t)},
$$

with

$$
\dot{\varphi}_{k}(t)=-\frac{E_{\mathrm{mb} ; k}(q)}{\hbar} ; \quad q=q(t) .
$$

Let us emphasize that both $\Psi_{\mathrm{mb}}^{\mathrm{ad}}(x, q, t)$ and $\Phi_{\mathrm{mb} ; k}(x ; q)$ play, in this discussion, a role of auxiliary reference functions [the ensemble $\left\{\Phi_{\mathrm{mb} ; k}(x ; q)\right\}$ can be treated as a set of basis wave functions] and should not be attributed any meaning of solutions of the collective motion problem at this stage; discussion of collective properties of interest in this article will follow the definition of the collective equation of motion, see Eqs. (38)-(40).

In this linear combination, the evolution of $\hat{H}_{\mathrm{mb}}^{\mathrm{ad}}(\hat{X} ; q)$ induced by varying $q$ is now represented by the $C_{k}(t)$ coefficients. We can add that the time enters this wave function in two fashions: by a rapid one in the exponentials $e^{i \varphi_{k}(t)}$ and a slow one in the $C_{k}(t)$ coefficients and in $q=q(t)$. By "slow" we mean that modifications of the first order in $t$ produce modification of $C_{k}(t)$ and $q(t)$ only at most at a second order.

To describe the collective motion in the spirit of the Bohr formulation, we introduce the nuclear collective Hamiltonian

$$
\hat{H}_{\mathrm{col}}=\hat{T}_{\mathrm{col}}+\hat{V}_{\mathrm{col}}(q) \text {. }
$$

The above expression of the collective Hamiltonian is formally independent of the preceding discussion; however, relations (4)-(7) will serve to construct in particular $\hat{T}_{\text {col }}$ in the spirit of the Bohr collective model. The associated Schrödinger equation governs the collective nuclear motion formally attributed to a new quantum structureless object here referred to as quanton; quanton energies are interpreted as the energies of the nuclear collective motion.

Collective potential energy in Eq. (8), in most frequent phenomenological realizations of the approach is constructed using the Strutinsky method. The collective kinetic energy operator is constructed using the notion of the collective inertia tensor. The latter is usually calculated using the generic perturbation-theory expression $[7,17,18]$, employing the equations of evolution of the $C_{k}(t)$ coefficients. The corresponding expression for the mass tensor components

$$
\begin{aligned}
B_{n m}(q)= & 2 \hbar^{2} \sum_{j=1}^{N}\left\langle\Phi_{\mathrm{mb} ; j}(x ; q)\left|\frac{\partial}{\partial q^{n}}\right| \Phi_{\mathrm{mb} ; 0}(x ; q)\right\rangle \\
& \times\left\langle\Phi_{\mathrm{mb} ; j}(x ; q)\left|\frac{\partial}{\partial q^{m}}\right| \Phi_{\mathrm{mb} ; 0}(x ; q)\right\rangle^{*} \\
& \times \frac{1}{E_{\mathrm{mb} ; j}(q)-E_{\mathrm{mb} ; 0}(q)}
\end{aligned}
$$

employs the auxiliary basis $\Phi_{\mathrm{mb} ; j}(x ; q)$ of Eq. (5). Above, $N$ is the number of wave functions from (5) retained for the model description of the collective properties via inertia tensor; it plays a role of a cutoff parameter of the method, usually selected in such a way that the results of interest do not depend on it in any significant manner. It has been demonstrated in Chapter 1 of Ref. [1] that this well-known expression can be significantly improved and in fact should be replaced by a new formula, whose justification is briefly presented below.

In order to obtain the nuclear-shape-dependent inertia tensor, we use the simultaneous validity of Eqs. (4) and (5) and the expansion (6). Introducing (6) into (4), we find the system of differential equations which govern the time evolution of $C_{k}(t)$

$$
\begin{aligned}
\dot{C}_{j}(t)= & -\sum_{n=0}^{d} \dot{q}^{n}(t) \sum_{k=1}^{N}\left\langle\Psi_{\mathrm{mb} ; j}[x ; q(t)]\left|\frac{\partial}{\partial q^{n}}\right| \Psi_{\mathrm{mb} ; k}[x ; q(t)]\right\rangle \\
& \times e^{i \varphi_{k j}(t)} C_{k}(t),
\end{aligned}
$$

where

$$
\varphi_{k j}(t)=\varphi_{k}(t)-\varphi_{j}(t)
$$

and

$$
\varphi_{k}(t)=-\frac{1}{\hbar} \int_{t_{0}}^{t} d t^{\prime} E_{\mathrm{mb} ; k}\left[q\left(t^{\prime}\right)\right] .
$$

Since one is primarily interested in the properties of the nuclear ground-state configurations, this system of equations 
is usually solved under the initial conditions assuming that at $t=t_{0}$ the auxiliary function $\Psi_{\mathrm{mb}}^{\mathrm{ad}}\left(x, q, t_{0}\right)$ corresponds to the $(j=0)$ basis state [4]:

$$
\begin{aligned}
\Psi_{\mathrm{mb}}^{\mathrm{ad}}\left(x, q, t_{0}\right) & =\Phi_{\mathrm{mb} ; 0}(x ; q): C_{0}\left(t_{0}\right)=1, \\
C_{j>0}\left(t_{0}\right) & =0, \quad \varphi_{j \geqslant 0}\left(t_{0}\right)=0 .
\end{aligned}
$$

According to the adiabatic theorem, the $(j=0)$ basis state varies slowly as a function of $q$ and it represents the lowest energy basis state also for $t<t_{0}$ and $t>t_{0}$ at least for a certain lapse of time. This implies that $C_{0}(t) \approx 1$ and $\left|C_{j>0}(t)\right| \ll$ $\left|C_{0}(t)\right|$. Thus for a not too long lapse of time, $\tau$ :

$$
\begin{aligned}
& C_{0}(t) \approx 1, \\
& \left|C_{j>0}(t)\right| \ll\left|C_{0}(t)\right|, \quad \text { where } t_{0}-\frac{\tau}{2} \leqslant t \leqslant t_{0}+\frac{\tau}{2} \\
& \dot{q}^{n}(t) \approx \dot{q}^{n}\left(t_{0}\right), \\
& \Phi_{\mathrm{mb} ; j \geqslant 0}[x ; q(t)] \approx \Phi_{\mathrm{mb} ; j \geqslant 0}\left[x ; q\left(t_{0}\right)\right],
\end{aligned}
$$

and the system of differential equations takes the form

$$
\begin{aligned}
\dot{C}_{j>0}(t) \approx & -e^{i \varphi_{0 j}(t)} \sum_{n=1}^{d} \dot{q}^{n}\left(t_{0}\right) \\
& \times\left\langle\Phi_{\mathrm{mb} ; j}\left[x ; q\left(t_{0}\right)\right]\left|\frac{\partial}{\partial q^{n}}\right| \Phi_{\mathrm{mb} ; 0}\left[x ; q\left(t_{0}\right)\right]\right\rangle,
\end{aligned}
$$

where

$$
\begin{aligned}
\varphi_{0 j}(t) & =\frac{1}{\hbar} \int_{t_{0}}^{t} d t^{\prime}\left\{E_{\mathrm{mb} ; j}[q(t)]-E_{\mathrm{mb} ; 0}[q(t)]\right\} \\
& \approx \frac{E_{\mathrm{mb} ; j}\left[q\left(t_{0}\right)\right]-E_{\mathrm{mb} ; 0}\left[q\left(t_{0}\right)\right]}{\hbar}\left(t-t_{0}\right) .
\end{aligned}
$$

Following the above linearity in terms of time, the time integration of the exponential can be performed explicitly

$$
\int_{t_{0}}^{t} d t^{\prime} e^{i \varphi_{0 j}\left(t^{\prime}\right)}=-\frac{i \hbar\left[e^{i \varphi_{0 j}(t)}-1\right]}{E_{\mathrm{mb} ; j}\left[q\left(t_{0}\right)\right]-E_{\mathrm{mb} ; 0}\left[q\left(t_{0}\right)\right]} .
$$

This result is at the origin of the difference between the present and the "traditional" derivation of the microscopic expression for collective inertia tensor. It implies in particular also a new form of the solutions of the considered system of differential equations:

$$
\begin{aligned}
C_{j>0}(t) \approx & \sum_{n=1}^{d} \frac{i \hbar\left[e^{i \varphi_{0 j}(t)}-1\right]}{E_{\mathrm{mb} ; j}\left[q\left(t_{0}\right)\right]-E_{\mathrm{mb} ; 0}\left[q\left(t_{0}\right)\right]} \dot{q}^{n}\left(t_{0}\right) \\
& \times\left\langle\Phi_{\mathrm{mb} ; j}\left[x ; q\left(t_{0}\right)\right]\left|\frac{\partial}{\partial q^{n}}\right| \Phi_{\mathrm{mb} ; 0}\left[x ; q\left(t_{0}\right)\right]\right\rangle .
\end{aligned}
$$

Let us remark in passing that in the traditional approach to solving the system of linear equations in Eq. (10) one chooses working with indefinite integrals (primitive functions) leading to the time-dependent factors $e^{i \varphi_{0 j}(t)}$ rather than $\left[e^{i \varphi_{0 j}(t)}-1\right]$ as in Eq. (19). The latter choice allows respecting the initial condition (13) rigorously, whereas the former leads to the disappearance of the contribution of these factors to the collective mass tensor altogether. Our choice leads to the presence of (generally rapidly) varying time-dependent factor which is time-averaged in the present realization of the adiabatic approximation.
Following the approach of Refs. [7,9], the total energy of the system is obtained with the initial conditions in Eq. (13) as the expected value in the form

$$
E_{\mathrm{tot}}(q, \dot{q}, t) \equiv\left\langle\Psi_{\mathrm{mb}}^{\mathrm{ad}}(x, q, t)\left|\hat{H}_{\mathrm{mb}}^{\mathrm{ad}}(\hat{x} ; q)\right| \Psi_{\mathrm{mb}}^{\mathrm{ad}}(x, q, t)\right\rangle
$$

which, with the help of Eqs. (5) and (6), gives

$E_{\mathrm{tot}}(q, \dot{q}, t)=E_{\mathrm{mb} ; 0}(q)+\sum_{j=1}^{N}\left|C_{j}(t)\right|^{2}\left[E_{\mathrm{mb} ; j}(q)-E_{\mathrm{mb} ; 0}(q)\right]$,

a relation applicable to the adiabatic, quasistationary motion for $t$ in the vicinity of $t_{0}$. It can be seen from Eq. (19) that $\left|C_{j}(t)\right|^{2}$ are bilinear forms of the collective velocities $\left\{\dot{q}^{n}\right\}$ and it follows that the second term above can be interpreted as an analog of classical kinetic energy. In the same context $E_{\mathrm{mb} ; 0}(q)$ is considered as potential energy of the system. Energy $E_{\text {tot }}(q, \dot{q}, t)$ depends on the individual-nucleonic motion via $C_{j}(t)$.

At this point comes the novelty of the present approach. We describe the collective motion in the presence of the adiabatic separation of the two timescales via averaging out explicitly the fast-fluctuating contributions over an averagingtime interval, say $\tau$, in a manner which is mathematically more rigorous than the one used in the literature. This interval should be sufficiently long to cover the individual fast fluctuations and short enough so that the slowly varying component can still be described as slowly moving. The actual value of $\tau$, playing a role of an adiabaticity parameter, allows us to control the relative contributions of the two types of motion via comparison with the experimental data, see below, and is expected to lie between $\tau_{\text {ind }}$ and $\tau_{\text {col }}$

$$
\tau_{\text {ind }} \ll 1 \times 10^{-22} \leqslant \tau \leqslant 1 \times 10^{-21} \mathrm{~s} \ll \tau_{\text {col }} .
$$

Time-averaging around any given instant $t_{0}$ leads to

$$
E_{\mathrm{col}}\left(q, \dot{q} ; t_{0}, \tau\right)=\frac{1}{\tau} \int_{-\frac{\tau}{2}}^{\frac{\tau}{2}} d t^{\prime} E_{\mathrm{tot}}\left(q, \dot{q}, t_{0}+t^{\prime}\right) .
$$

Using the explicit time dependence in the $C_{j}$ coefficients, Eq. (19), together with relation (21), the time integration can be obtained exactly in the form of the following compact expression:

$$
\begin{aligned}
\aleph_{0 j}\left[q\left(t_{0}\right) ; \tau\right] & \equiv \frac{1}{\tau} \int_{-\frac{\tau}{2}}^{\frac{\tau}{2}} d t^{\prime}\left|e^{i \varphi_{0 j}\left(t_{0}+t^{\prime}\right)}-1\right|^{2} \\
& =2-2 \operatorname{sinc}\left(\frac{E_{\mathrm{mb} ; j}\left[q\left(t_{0}\right)\right]-E_{\mathrm{mb} ; 0}\left[q\left(t_{0}\right)\right]}{2 \hbar} \tau\right),
\end{aligned}
$$

where $\operatorname{sinc}(x) \equiv \frac{\sin (x)}{x}$. This leads to

$$
\begin{aligned}
E_{\mathrm{col}}\left(q, \dot{q} ; t_{0}, \tau\right)= & E_{\mathrm{mb} ; 0}\left[q\left(t_{0}\right)\right]+\sum_{m=1}^{d} \sum_{n=1}^{d} \frac{1}{2} B_{n m}\left[q\left(t_{0}\right) ; \tau\right] \\
& \times \dot{q}^{n}\left(t_{0}\right) \dot{q}^{m}\left(t_{0}\right),
\end{aligned}
$$




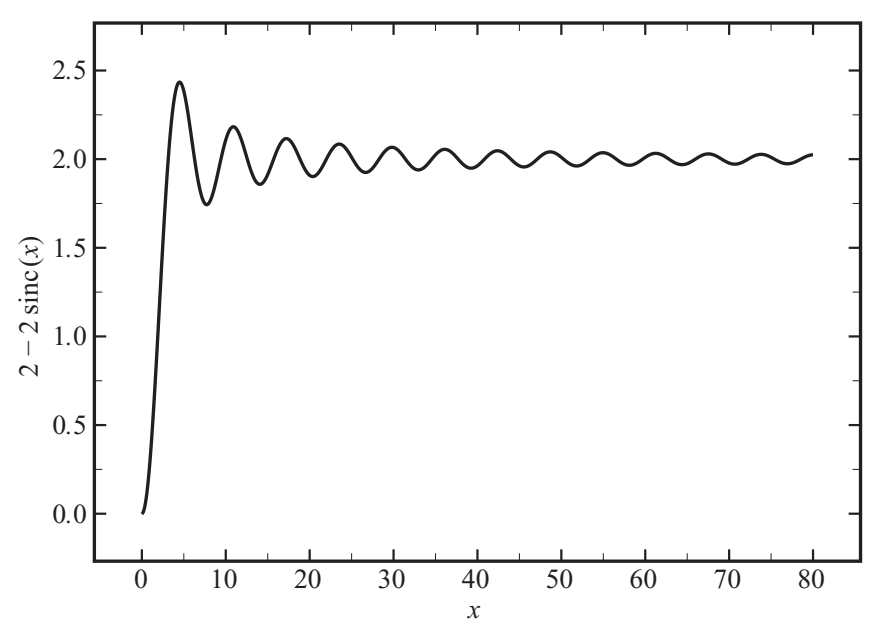

FIG. 1. Universal function $\aleph_{0 j}(q ; \tau)$ referred to as temporizer; $x \equiv \frac{E_{\mathrm{mb} ; j}(q)-E_{\mathrm{mb} ; 0}(q)}{2 \hbar} \tau$.

with the modified definition of the mass tensor

$$
\begin{aligned}
B_{n m}(q ; \tau)= & 2 \hbar^{2} \sum_{j=1}^{N}\left\langle\Phi_{\mathrm{mb} ; j}(x ; q)\left|\frac{\partial}{\partial q^{n}}\right| \Phi_{\mathrm{mb} ; 0}(x ; q)\right\rangle \\
& \times\left\langle\Phi_{\mathrm{mb} ; j}(x ; q)\left|\frac{\partial}{\partial q^{m}}\right| \Phi_{\mathrm{mb} ; 0}(x ; q)\right\rangle^{*} \\
& \times \frac{\aleph_{0 j}(q ; \tau)}{E_{\mathrm{mb} ; j}(q)-E_{\mathrm{mb} ; 0}(q)} .
\end{aligned}
$$

The above expression differs from the usual one found in the literature, cf. Eq. (9), by the presence of an extra factor, $\aleph_{0 j}(q ; \tau)$. To stress that the presence of this factor originates from the description of the two timescales we refer to it as temporizer, see Ref. [1], p. 30. Function $\aleph_{0 j}(q ; \tau)$ carries certain features of universality as represented in Fig. 1. When distinction between collective and individual timescales cannot be made, i.e., $\tau \rightarrow 0$, we have $\aleph_{0 j}(q ; \tau) \rightarrow 0$ (as Fig. 1 shows) there is no more time-averaging possible, and the inertia tensor vanishes, see Eq. (26). This limit creates no intrinsic conflicts because with no time-averaging, one can introduce neither collective Schrödinger equation, nor quanton, nor collective inertia.

Let us mention that the traditional expression for the mass tensor reduces, when imposing the condition of the translational motion, to nuclear mass whereas the present approach does not give the same result under the same conditions. We believe that the main motivation for the microscopic method of calculation of the nuclear mass tensor as functional of nuclear shape is both to provide the link between collective inertia with the microscopic (single-nucleonic) degrees of freedom and an acceptable empirical correspondence with experimental data when inserting the corresponding mass tensor to the collective Schrödinger equation. If particularly motivated, one could use the presence of the empirical factor $\tau$ and adjust it to the desired asymptotic condition.

In the traditional realizations of the discussed theory, the collective characteristics of the system, $E_{\mathrm{mb} ; 0}(q)$ and $B_{n m}(q ; \tau)$, are calculated after solving Eq. (5) for different fixed values of $q$, usually employing the nuclear mean-field theory: $\hat{H}_{\mathrm{mb}}^{\mathrm{ad}}(\hat{X} ; q) \rightarrow \hat{H}_{\mathrm{mf}}(\hat{X} ; q)$. We use the phenomenological deformed Woods-Saxon ${ }^{1}$ mean-field approximation $\hat{H}_{\mathrm{mf}}(\hat{x} ; q) \rightarrow \hat{H}_{\mathrm{WS}}(\hat{x} ; q \rightarrow \alpha)$, where the collective degrees of freedom are defined as the coefficients of the nuclear surface $R(\theta, \phi ; \alpha)$ expansion in terms of spherical harmonics

$$
R(\theta, \phi ; \alpha)=R_{0} c(\alpha)\left(1+\sum_{\lambda=2}^{\infty} \sum_{\mu=-\lambda}^{\lambda} \alpha_{\lambda}{ }^{\mu} Y_{\lambda \mu}(\theta, \phi)\right),
$$

where $\alpha \equiv\left\{\alpha_{\lambda}{ }^{\mu}\right\}$.

From now on we assume that the Woods-Saxon singleparticle energies, $E_{\mathrm{sp} ; i}(\alpha)$, and wave functions, $\Phi_{\mathrm{sp} ; i}\left(x_{i} ; \alpha\right)$, are known and that the collective total nuclear energy in Eq. (21) has been calculated accordingly using the standard phenomenological Strutinsky method. To proceed, we specify the nature of excited states in the denominators in (26) adapted to the mean-field approximation assuming as usual that only the one-particle-one-hole bound-state energies (or two quasiparticle excitations in the case of the BCS pairing) are retained

$$
\Phi_{\mathrm{mb} ; j}(x ; q) \rightarrow \Phi_{\mathrm{mb} ; i, k}(x ; q)=\hat{c}_{k}^{+} \hat{c}_{i} \Phi_{\mathrm{mb} ; 0}(x ; q) .
$$

Above the single index $j$ needs to be replaced by two nonvanishing indices $k \in \mathbb{Z}_{\mathrm{p}}$ and $i \in \mathbb{Z}_{\mathrm{h}}$ specifying particle-hole excitations and it follows that

$$
E_{\mathrm{mb} ; j}(q)-E_{\mathrm{mb} ; 0}(q) \rightarrow E_{\mathrm{sp} ; k}(q)-E_{\mathrm{sp} ; i}(q)
$$

and

$$
\begin{aligned}
& \left\langle\Phi_{\mathrm{mb} ; j}(x ; q)\left|\frac{\partial}{\partial q^{n}}\right| \Phi_{\mathrm{mb} ; 0}(x ; q)\right\rangle \\
& \rightarrow\left\langle\Phi_{\mathrm{sp} ; k}\left(x_{k} ; q\right)\left|\frac{\partial}{\partial q^{n}}\right| \Phi_{\mathrm{sp} ; i}\left(x_{i} ; q\right)\right\rangle .
\end{aligned}
$$

The expression of the mass tensor takes a new form:

$$
\begin{aligned}
B_{n m}(q ; \tau)= & 2 \hbar^{2} \sum_{i \in \mathbb{Z}_{\mathrm{h}}} \sum_{k \in \mathbb{Z}_{\mathrm{p}}} \frac{\aleph_{0(i, k)}(q ; \tau)}{E_{\mathrm{sp} ; k}(q)-E_{\mathrm{sp} ; i}(q)} \\
& \times\left\langle\Phi_{\mathrm{sp} ; k}\left(x_{k} ; q\right)\left|\frac{\partial}{\partial q^{n}}\right| \Phi_{\mathrm{sp} ; i}\left(x_{i} ; q\right)\right\rangle \\
& \times\left\langle\Phi_{\mathrm{sp} ; i}\left(x_{i} ; q\right)\left|\frac{\partial}{\partial q^{m}}\right| \Phi_{\mathrm{sp} ; k}\left(x_{k} ; q\right)\right\rangle .
\end{aligned}
$$

Even though the above expression gives a compact representation of the essential dependencies without pairing, all the implied detailed and computer-programmable expressions have been obtained in Chapter 3 of Ref. [1].

Let us comment in passing about the singularities due to the level crossings in the denominators of the mass tensor expressions. Even though Eq. (31) contains none, since $\aleph_{0(i, k)}(q ; \tau) \rightarrow 0$ when $E_{\mathrm{sp} ; k}(q) \rightarrow E_{\mathrm{sp} ; i}(q)$, some authors choose employing the theorem of Hellmann-FeynmanEpstein and express the matrix elements of $\frac{\partial}{\partial q^{n}}$ with the help

\footnotetext{
${ }^{1}$ Here, the Woods-Saxon mean-field approach is supplemented with the BCS pairing extension.
} 
of the Hamiltonian as follows:

$$
\begin{aligned}
& \left\langle\Phi_{\mathrm{sp} ; k}(x ; q)\left|\frac{\partial}{\partial q^{n}}\right| \Phi_{\mathrm{sp} ; i}(x ; q)\right\rangle=\frac{-1}{E_{\mathrm{sp} ; k}(q)-E_{\mathrm{sp} ; i}(q)} \\
& \quad \times\left\langle\Phi_{\mathrm{sp} ; k}(x ; q)\left|\frac{\partial \hat{H}_{\mathrm{mf}}}{\partial q^{n}}\right| \Phi_{\mathrm{sp} ; i}(x ; q)\right\rangle .
\end{aligned}
$$

The matrix element on the left-hand side is given by integrals of the products of polynomial expressions and exponential factors guaranteeing their convergence $\forall k, i$. If the Hamiltonian in question obeys the Hellmann-Feynman-Epstein theorem for all eigenvalues including the degenerate ones, the divergencies are compensated for by the corresponding matrix elements of $\frac{\partial \hat{H}_{\mathrm{mf}}}{\partial q^{n}}$. In the opposite case, transformation in Eq. (32) loses its validity and transforming the form (31) using (32) is not permitted; the mass tensor expression remains nonsingular.

We will schematize briefly the formalism leading to the final Schrödinger equation in the curvilinear space spanned by the microscopically calculated inertia tensor. By solving such an equation we are able to test the theory predictions with selected experimental data.

In what follows we postulate that the collective space is Riemanian and its metric is given by the mass tensor. In classical (i.e., before quantizing in the curvilinear space) terms, collective potential energy is fixed by the Strutinsky algorithm

$$
V_{\mathrm{col}}(q)=E_{\mathrm{mb} ; 0}(q)
$$

whereas the collective kinetic energy reads

$$
T_{\mathrm{col}}(q, \dot{q} ; \tau)=\sum_{m, n=1}^{d} \frac{1}{2} B_{n m}(q ; \tau) \dot{q}^{n} \dot{q}^{m},
$$

leading to the associated Lagrangian

$$
L_{\mathrm{col}}(q, \dot{q} ; \tau)=\sum_{m, n=1}^{d} \frac{1}{2} B_{n m}(q ; \tau) \dot{q}^{n} \dot{q}^{m}-E_{\mathrm{mb} ; 0}(q) .
$$

This form leads to the collective Schrödinger equation by quantization of the associated Hamilton-Jacobi equation. We employ the usual quantization rules in the spatial representation, the collective position operator being simply the collective position

$$
\hat{q}^{n}=q^{n} \hat{\mathbb{1}}
$$

and the collective generalized momentum operator proportional to the covariant derivative ${ }^{2}$

$$
\hat{p}_{\mathrm{col}, n}=-i \hbar \nabla_{n}
$$

\footnotetext{
${ }^{2}$ Let us remind the reader that the covariant derivative of a scalar can be identified with the corresponding partial derivative, whereas the covariant derivative of a covariant vector is$$
\nabla_{n} p_{m}=\frac{\partial p_{m}}{\partial q^{n}}-\sum_{h=1}^{d} \Gamma_{m n}^{h} p_{h},
$$

where $\Gamma$ stands for the Christoffel symbol.
}

TABLE I. Calculated energies in $\mathrm{keV}$ and reduced transition probabilities in Weisskopf units for the $I^{\pi}=3_{1}^{-}, 2_{1}^{+}$, and $0_{2}^{+}$states in ${ }^{208} \mathrm{~Pb}$ for increasing $\tau$, Eq. (22). The penultimate line shows the results for the "traditional" way of mass tensor calculations like, e.g., in Ref. [7]. The last line shows the experimental data in Ref. [20].

\begin{tabular}{lccccc}
\hline \hline$\tau / 10^{-22} \mathrm{~s}$ & $E\left(3_{1}^{-}\right)$ & $B(E 3)$ & $E\left(2_{1}^{+}\right)$ & $B(E 2)$ & $E\left(0_{2}^{+}\right)$ \\
\hline 1.00 & 9365 & 158 & 9221 & 78.4 & 9282 \\
3.33 & 3229 & 55.1 & 5047 & 23.8 & 5926 \\
$\mathbf{4 . 5 2}$ & $\mathbf{2 6 1 4}$ & 45.4 & 4223 & 19.8 & 4786 \\
$\mathbf{4 . 9 4}$ & 2444 & 43.0 & $\mathbf{4 0 8 5}$ & 19.0 & 4646 \\
5.00 & 2423 & 42.7 & 4075 & 18.9 & 4634 \\
6.67 & 2017 & 37.0 & 3943 & 16.9 & 4692 \\
$\mathbf{7 . 0 0}$ & 1967 & 36.3 & 3947 & 16.6 & $\mathbf{4 8 6 8}$ \\
10.0 & 1735 & 32.8 & 3632 & 13.4 & 4305 \\
$\aleph=1$ & 2690 & 45.0 & 4078 & 40.7 & 5484 \\
Expt. & $\mathbf{2 6 1 4}$ & 33.8 & $\mathbf{4 0 8 5}$ & 8.4 & $\mathbf{4 8 6 8}$ \\
\hline \hline
\end{tabular}

It follows that the collective energy operator is

$$
\hat{H}_{\mathrm{col}}=-\frac{\hbar^{2}}{2} \Delta+E_{\mathrm{mb} ; 0}(q)
$$

with the Riemann-space Laplace operator given by

$$
\begin{aligned}
\Delta \Psi_{\mathrm{col} ; i} & =\sum_{m, n=1}^{d} B^{n m} \nabla_{n} \nabla_{m} \Psi_{\mathrm{col} ; i} \\
& =\sum_{m, n=1}^{d} \frac{1}{\sqrt{|B|}} \frac{\partial}{\partial q^{n}}\left(\sqrt{|B|} B^{n m} \frac{\partial \Psi_{\mathrm{col} ; i}}{\partial q^{m}}\right) .
\end{aligned}
$$

As an illustration, the formalism presented so far was applied to calculate the mass tensor $B_{m n}$ of Eq. (31) and the total Strutinsky energy $E_{\mathrm{mb} ; 0}$ of Eq. (21) in the three-dimensional space of variables $\left\{\alpha_{2}{ }^{0}, \alpha_{2}{ }^{2}, \alpha_{3}{ }^{0}\right\}$ for the ${ }^{208} \mathrm{~Pb}$ nucleus using the so-called Woods-Saxon universal mean-field approach [19]. The corresponding Schrödinger equation with Hamiltonian (38) was solved numerically with an adapted algorithm taking into account that in the curvilinear spaces we have to deal with a nonorthogonal basis. The resulting energies of the collective $3_{1}^{-}, 2_{1}^{+}$, and $0_{2}^{+}$excitations and the related $B(E \lambda)$ reduced transition probabilities were obtained and compared with experiment for varying $\tau$ parameter, cf. Eq. (22). The results are shown in Table I.

Results in Table I illustrate a variation of the predictions for the collective energies with increasing $\tau$. Comparison shows that selecting $\tau=5 \times 10^{-22} \mathrm{~s}$ [the middle of the interval in Eq. (22)] leads to the differences between the theory predictions and experimental results for the energies of the $I^{\pi}=$ $3_{1}^{-}, 2_{1}^{+}$, and $0_{2}^{+}$states of $7 \%, 0.25 \%$, and $5 \%$, respectively. This shows the overall consistency of the presented approach since the discrepancies of this order are not surprising when employing first-order perturbation approach. Consequently $\tau=5 \times 10^{-22} \mathrm{~s}$ can be seen as a good starting choice, whereas more systematic comparison for several nuclei (work in progress) would allow a more detailed analysis.

The transition probabilities illustrated in the Table I are obtained with the help of the transition matrix elements of the multipole-moment operators between the solutions-initial 
$\Psi_{\mathrm{col} ; i}$ and final $\Psi_{\mathrm{col} ; f}$ states of the transition-of the collective Schrödinger equation; we find

$$
\begin{aligned}
\left\langle Q_{\lambda \mu}\right\rangle_{i f} & =\left\langle\Psi_{\mathrm{col} ; i}\left|\hat{Q}_{\lambda \mu}\right| \Psi_{\mathrm{col} ; f}\right\rangle \\
& =\int_{\mathbb{R}^{3}} d \alpha_{2}{ }^{0} d \alpha_{2}{ }^{2} d \alpha_{3}{ }^{0} \Psi_{\mathrm{col} ; i}^{*}(\alpha) Q_{\lambda \mu} \Psi_{\mathrm{col} ; f}(\alpha) .
\end{aligned}
$$

The multipole moment operators can be expressed for not too large values of the coordinates $\alpha_{\lambda}{ }^{\mu}$ using the uniform chargedensity approximation as

$$
\begin{aligned}
Q_{\lambda \mu} & =\int_{V} d^{3} r \rho r^{\lambda} Y_{\lambda \mu}^{*} \\
& =\frac{3 Z e}{4 \pi R_{0}^{3}} \int_{0}^{2 \pi} d \phi \int_{0}^{\pi} d \theta \sin \theta Y_{\lambda \mu}^{*}(\theta, \phi) \int_{0}^{R(\theta, \phi ; \alpha)} d r r^{\lambda+2},
\end{aligned}
$$

and it follows that for $\lambda \leqslant 3$ we have

$$
\begin{aligned}
Q_{20}= & \frac{3 Z e R_{0}^{2}}{4 \pi \sqrt{\pi}}\left[\sqrt{\pi} \alpha_{2}{ }^{0}+\frac{2 \sqrt{5}}{7}\left(\alpha_{2}{ }^{0}\right)^{2}\right. \\
& \left.-\frac{4 \sqrt{5}}{7}\left(\alpha_{2}{ }^{2}\right)^{2}+\frac{4}{3 \sqrt{5}}\left(\alpha_{3}{ }^{0}\right)^{2}\right], \\
Q_{2 \pm 2}= & \frac{3 Z e R_{0}^{2}}{4 \pi \sqrt{\pi}}\left[\sqrt{\pi} \alpha_{2}{ }^{2}-\frac{4 \sqrt{5}}{7} \alpha_{2}{ }^{0} \alpha_{2}{ }^{2}\right], \\
Q_{30}= & \frac{3 Z e R_{0}^{3}}{4 \pi \sqrt{\pi}}\left[\sqrt{\pi} \alpha_{3}{ }^{0}+\frac{2 \sqrt{5}}{3} \alpha_{2}{ }^{0} \alpha_{3}{ }^{0}\right], \\
Q_{3 \pm 2}= & \frac{3 Z e R_{0}^{3}}{4 \pi \sqrt{\pi}}\left[\sqrt{\pi} \alpha_{3}{ }^{2}-\frac{5}{3} \alpha_{2}{ }^{2} \alpha_{3}{ }^{0}\right],
\end{aligned}
$$

the other $\{\lambda, \mu\}$ combinations vanishing. The reduced transition probabilities are given as usual by

$$
B\left(E \lambda, \Psi_{\mathrm{col} ; i} \rightarrow \Psi_{\mathrm{col} ; f}\right)=\sum_{\mu=-\lambda}^{\lambda}\left|\left\langle\Psi_{\mathrm{col} ; i}\left|\hat{Q}_{\lambda \mu}\right| \Psi_{\mathrm{col} ; f}\right\rangle\right|^{2} .
$$

For completeness, the Weisskopf units (Wu) are defined by

$$
B_{\mathrm{W}}(E \lambda)=\frac{e^{2} R_{0}^{2 \lambda}}{4 \pi}\left(\frac{3}{\lambda+3}\right)^{2} .
$$

Selection of the ${ }^{208} \mathrm{~Pb}$ for the present comparison was motivated by the existing experimental information about the low-lying collective vibrational states mentioned as well as by the fact that for this doubly magic nucleus the pairing correlations are expected to be dominantly absent for the small-amplitude vibrations around the spherical shape. The latter argument allows us to ignore the pairing correlations, to an approximation, thus limiting the extra parametric freedom (and parametric uncertainties) in terms of the proton and neutron pairing strengths.

Comparison of the results in Table I indicates that the main idea of this article, i.e., the possibility of introducing directly the microscopic two timescales for the individual (rapid) and collective (slow) nuclear motions together with a parametric measure of their interplay with the help of a single parameter $\tau$, ensures the close correspondence between the calculated energies and the experimental data in the physically justified $\tau$ interval. Let us emphasize that the new approach is free from the singularities of the traditional one in terms of the crossings of the levels in the denominators of the mass tensor formula of Eq. (31).

Let us remark that, as seen from Table I, the $B(E 2)$ calculated according to the traditional algorithm, i.e., corresponding to $\aleph=1$, can be viewed as particularly large, almost double the value calculated according to the new algorithm with the reference choice of $\tau=5 \times 10^{-22} \mathrm{~s}$. In calculating the reduced transition probabilities the decisive role for the final result is played by the integral expression (48) given by the integrals of the collective wave functions-solutions of the collective Schrödinger equation involving relations (38)-(40). The main impact on the discussed difference originates in our case from the uncompensated singularities caused by the level crossings in the denominators of Eq. (31) in the case of the traditional approach.

Let us mention furthermore that the nuclear potential energies calculated using the Strutinsky method depend, among others, on the macroscopic energy term whose contribution to the energy curvature close to the equilibrium is not controlled. Indeed, no parameters of the macroscopic energy term are fitted to, e.g., collective excitations but primarily to the nuclear masses and fission barriers. This element of the phenomenological theory can be used to optimize further the agreement with the experimental data which can already be considered satisfactory.

J.D. performed part of this work within the EMPIR Project 15SIB10 MetroBeta. This project has received funding from the EMPIR program cofinanced by the Participating States and from the European Union Horizon 2020 research and innovation program. J.D. acknowledges partial support by the Polish National Science Centre (PL) under Contract No. 2016/21/B/ST2/01227.
[1] D. Rouvel, Ph.D. thesis, University of Strasbourg, 2014.

[2] J. Dudek, W. Dudek, E. Ruchowska, and J. Skalski, Z. Phys. A 294, 341 (1980).

[3] N. Schunck and L. M. Robledo, Rep. Prog. Phys. 79, 116301 (2016).

[4] P. Ring and P. Schuck, The Nuclear Many-Body Problem (Springer, New York, 2000).
[5] P.-G. Reinhard and K. Goeke, Rep. Prog. Phys. 50, 1 (1987).

[6] A. Baran, J. A. Sheikh, J. Dobaczewski, W. Nazarewicz, and A. Staszczak, Phys. Rev. C 84, 054321 (2011).

[7] M. Brack, J. Damgaard, A. S. Jensen, H. C. Pauli, V. M. Strutinsky, and C. Y. Wong, Rev. Mod. Phys. 44, 320 (1972).

[8] R. E. Peierls and J. Yoccoz, Proc. Phys. Soc. A 70, 381 (1957).

[9] S. T. Belyaev, Mat. Fys. Medd. Dan. Vid. Selsk. 31 (1959). 
[10] D. J. Rowe and R. Bassermann, Nucl. Phys. A 220, 404 (1974).

[11] F. Villars, Nucl. Phys. A 285, 269 (1977).

[12] B. Slavov, V. I. Dimitrov, K. Göke, F. Grümmer, and P.-G. Reinhard, Nucl. Phys. A 454, 392 (1986).

[13] N. Hinohara, T. Nakatsukasa, M. Matsuo, and K. Matsuyanagi, Prog. Theor. Phys. 119, 59 (2008).

[14] L. Próchniak and S. G. Rohoziński, J. Phys. G: Nucl. Part. Phys. 36, 123101 (2009).

[15] M. Born and V. A. Fock, Z. Physik A 51, 165 (1928).

[16] T. Kato, J. Phys. Soc. Jpn. 5, 435 (1950).

[17] H. J. Krappe and K. Pomorski, Theory of Nuclear Fission (Springer-Verlag, Berlin, 2012).
[18] W. Greiner and J. A. Maruhn, Nuclear Models (Springer-Verlag, Berlin, 1996).

[19] The universal Woods-Saxon Hamiltonian and associated socalled universal parametrization were developed in a series of articles: J. Dudek and T. Werner, J. Phys. G: Nucl. Phys. 4, 1543 (1978); J. Dudek, A. Majhofer, J. Skalski, T. Werner, S. Ćwiok, and W. Nazarewicz, ibid. 5, 1359 (1979); J. Dudek, W. Nazarewicz, and T. Werner, Nucl. Phys. A 341, 253 (1980); J. Dudek, Z. Szymański, and T. Werner, Phys. Rev. C 23, 920 (1981); and has been summarized in S. Ćwiok, J. Dudek, W. Nazarewicz, J. Skalski, and T. Werner, Comput. Phys. Commun. 46, 379 (1987).

[20] ENSDF Database (2018). 